\title{
SUFFICIENCY OF MCMULLEN'S CONDITIONS FOR $f$-VECTORS OF SIMPLICIAL POLYTOPES
}

\author{
BY LOUIS J. BILLERA ${ }^{1}$ AND CARL W. LEE ${ }^{2}$
}

For convex $d$-polytope $P$ let $f_{i}(P)$ equal the number of faces of $P$ of dimension $i, 0 \leqslant i \leqslant d-1 . f(P)=\left(f_{0}(P), \ldots, f_{d-1}(P)\right)$ is called the $f$-vector of $P$. An important combinatorial problem is the characterization of the class of all $f$-vectors of polytopes, and in particular of simplicial polytopes (i.e. those for which each facet is a simplex). McMullen in [5] conjectures a set of necessary and sufficient conditions for $\left(f_{0}, \ldots, f_{d-1}\right)$ to be the $f$-vector of a simplicial $d$-polytope and proves this conjecture in the case of polytopes with few vertices. We sketch here a proof of the sufficiency ${ }^{3}$ of these conditions, and derive in a related way a general solution to an upper bound problem posed by Klee.

The $f$-vectors of simplicial $d$-polytopes satisfy the Dehn-Sommerville equations

$$
\sum_{i=j}^{d-1}(-1)^{i}\left(\begin{array}{l}
i+1 \\
j+1
\end{array}\right) f_{i}(P)=(-1)^{d-1} f_{j}(P), \quad-1 \leqslant j \leqslant d-1,
$$

where we put $f_{-1}(P)=1$. As in $\left[6\right.$, p. 170], for $d$-vector $f=\left(f_{0}, \ldots, f_{d-1}\right)$ and integer $e \geqslant d$ let

$$
g_{j}^{(e)}(f)=h_{j+1}^{(e)}(f)=\sum_{i=-1}^{j}(-1)^{j-i}\left(\begin{array}{l}
e-i-1 \\
e-j-1
\end{array}\right) f_{i}, \quad-1 \leqslant j \leqslant e-1,
$$

with the convention that $f_{-1}=1$ and $f_{i}=0$ for $i<-1$ or $i>d-1$. We note here that these relations are invertible, allowing us to express the $f_{i}$ as nonnegative linear combinations of the $h_{j}^{(e)}(f)$. The Dehn-Sommerville equations for $f$ are, for any $e \geqslant d$, equivalent to $g_{i}^{(e)}(f)=(-1)^{e-d} g_{e-i-2}^{(e)}(f),-1 \leqslant i \leqslant[e / 2]-1$. Let $h$ and $i$ be positive integers. Then $h$ can be written uniquely as

Received by the editors July 18, 1979.

1980 Mathematics Subject Classification. Primary 52A25; Secondary 05A15, 05A19, $05 \mathrm{~A} 20,90 \mathrm{C05}, 13 \mathrm{H} 10$. complex.

Key words and phrases. Convex polytope, $f$-vector, 0 -sequence, shelling, simplicial

${ }^{1}$ Supported in part by NSF grant MCS77-28392 and ONR contract N00014-75-C0678.

${ }^{2}$ Supported, in addition, by an NSF Graduate Fellowship.

${ }^{3}$ ADDED IN PROOF. R. Stanley has proved necessity since this was written. 


$$
h=\left(\begin{array}{c}
a_{i} \\
i
\end{array}\right)+\left(\begin{array}{c}
a_{i-1} \\
i-1
\end{array}\right)+\cdots+\left(\begin{array}{c}
a_{j} \\
j
\end{array}\right)
$$

where $a_{i}>a_{i-1}>\cdots>a_{j} \geqslant j \geqslant 1$. Following McMullen put

$$
h^{\langle i\rangle}=\left(\begin{array}{c}
a_{i}+1 \\
i+1
\end{array}\right)+\left(\begin{array}{c}
a_{i-1}+1 \\
i
\end{array}\right)+\cdots+\left(\begin{array}{c}
a_{j}+1 \\
j+1
\end{array}\right)
$$

and define $0^{\langle i\rangle}=0$. McMullen conjectured $\left([5],\left[6\right.\right.$, p. 179]) that $\left(f_{0}, \ldots\right.$, $\left.f_{d-1}\right)$ is the $f$-vector of a simplicial $d$-polytope if and only if the following three conditions hold:

$$
\begin{aligned}
& g_{i}^{(d+1)}(f)=-g_{d-i-1}^{(d+1)}(f), \quad-1 \leqslant i \leqslant[1 / 2(d+1)]-1, \\
& g_{i}^{(d+1)}(f) \geqslant 0, \quad 0 \leqslant i \leqslant n-1, \\
& g_{i}^{(d+1)}(f) \leqslant\left(g_{i-1}^{(d+1)}(f)\right)^{\langle i\rangle}, \quad 1 \leqslant i \leqslant n-1,
\end{aligned}
$$

where $n=[d / 2]$. Condition (1) is just the set of Dehn-Sommerville equations, the conjectured necessity of (2) is known as the Generalized Lower Bound Conjecture ([7], [6, p. 178]).

We will sketch a proof of the following

THEOREM 1. If $f=\left(f_{0}, \ldots, f_{d-1}\right)$ satisfies (1), (2), and (3) above, then $f=f(P)$ for some simplicial d-polytope $P$.

The case $d<2$ is easily dispensed with, so assume $d \geqslant 2$. For finite ( $d-$ 1)-dimensional simplicial complex $\Sigma$ let $|\Sigma|$ denote the underlying topological space of $\Sigma . f(\Sigma)=\left(f_{0}(\Sigma), \ldots, f_{d-1}(\Sigma)\right)$ is the $f$-vector of $\Sigma$, where $f_{i}(\Sigma)$ is the number of $i$-dimensional simplices in $\Sigma$. For $e \geqslant d$ write $h^{(e)}(\Sigma)$ for $h^{(e)}(f(\Sigma))$. We call $h^{(d)}(\Sigma)$ the $h$-vector of $\Sigma$. This is equivalent to Stanley's $h$-vector of [9]. If $|\Sigma|$ is a $(d-1)$-sphere then the Dehn-Sommerville equations hold (see, for example, Grünbaum [1, p. 152]). If $|\Sigma|$ is a $d$-ball then $\partial|\Sigma|$ is a $(d-1)$-sphere with associated complex $\partial \Sigma .|\Delta|$ is then a $d$-sphere, where $\Delta=$ $\Sigma \cup v \cdot \partial \Sigma$. It can be shown that $h_{i}^{(d+1)}(\Delta)=h_{i}^{(d+1)}(\Sigma)+h_{i-1}^{(d)}(\partial \Sigma), 0 \leqslant i \leqslant$ $d+1$ (where we take $h_{-1}^{(d)}(\partial \Sigma)=0$ ). The Dehn-Sommerville equations for $\Delta$ and $\partial \Sigma$ allow us to solve for $h_{i}^{(e)}(\partial \Sigma)$ in terms of $h_{j}^{(d+1)}(\Sigma)$. In particular [7]

$$
h_{i}^{(d+1)}(\partial \Sigma)=h_{i}^{(d+1)}(\Sigma)-h_{d+1-i}^{(d+1)}(\Sigma), \quad 0 \leqslant i \leqslant[1 / 2(d+1)] .
$$

A nonvoid set $M$ of monomials $Y_{1}^{a_{1}} \cdots Y_{s}^{a_{s}}$ is said to be an order ideal of monomials if whenever $m_{1} \in M$ and $m_{2} \mid m_{1}$ then $m_{2} \in M$. Let $\Phi$ be the set of all monomials in the variables $Y_{1}, \ldots, Y_{s}$. Give the elements of $\Phi$ the lexicographic linear order $<$ induced by $Y_{1}<\cdots<Y_{s}$. A finite or infinite sequence $(H(0), H(1), \cdots)$ of nonnegative integers is said to be an 0 -sequence 
if there exists an order ideal $M$ of monomials in the variables $Y_{1}, \ldots, Y_{s}$ with each $\operatorname{deg} Y_{i}=1$ such that $H(i)=\operatorname{card}\{m \in M: \operatorname{deg} m=i\}$. Stanley in [10] gives the following

THEOREM. Let $H: \mathbf{N} \rightarrow \mathbf{N}$. The following statements are equivalent:

(i) $(H(0), H(1), \cdots)$ is an 0 -sequence.

(ii) $H(0)=1$ and for all $i \geqslant 1, H(i+1) \leqslant H(i)^{\langle i\rangle}$.

(iii) Let $s=H(1)$ and for each $i \geqslant 0$ let $M_{i}$ be the first (in the ordering above) $H(i)$ monomials of degree $i$ in the variables $Y_{1}, \ldots, Y_{s}$. Define $M=\bigcup_{i \geqslant 0} M_{i}$. Then $M$ is an order ideal of monomials. Call $M$ the lexicographic order ideal of monomials associated with $(H(0), H(1), \cdots)$.

IdEA of PRoOf of THEOREM 1. If $\left(f_{0}, \ldots, f_{d-1}\right)$ satisfies (1), (2), and (3), then by the above theorem $(H(0), \ldots, H(d+1))$ is an 0 -sequence, where $H(i)=h_{i}^{(d+1)}(f)$ for $0 \leqslant i \leqslant n$ and $H(i)=0$ for $n+1 \leqslant i \leqslant d+1$. A simplicial complex $\Sigma$ is constructed by choosing as its maximal simplices certain $(d+$ 1)-sets from a $\nu$-set, where $\nu=H(1)+d+1$, such that $\Sigma$ is shellable in the sense of [9] and such that $(H(0), \ldots, H(d+1))$ is its $h$-vector. It is then shown that $\Sigma$ is the complex associated with a shellable proper collection $B$ of facets of the cyclic polytope $C(\nu, d+1)$, implying that $|\Sigma|$ is a $d$-ball. $\partial|\Sigma|$ is then a $(d-1)$-sphere with associated complex $\partial \Sigma$. From $H(i)=0, n+1 \leqslant i$ $\leqslant d+1$, it can be concluded that $h_{i}^{(d+1)}(\partial \Sigma)=h_{i}^{(d+1)}(f), 0 \leqslant i \leqslant n$. This and the Dehn-Sommerville equations for $\partial \Sigma$ yield $h^{(d+1)}(\partial \Sigma)=h^{(d+1)}(f)$, whence we conclude $f=f(\Sigma)$. Next, with an appropriate realization of $C(\nu, d+1)$ in $\mathbf{R}^{d+1}$, a point $z \in \mathbf{R}^{d+1}$ can be found such that $z$ is beyond those facets of $C(\nu, d+1)$ that are in $B$ and beneath the rest. Then the vertex figure $P$ of $z$ in $\operatorname{conv}(C(\nu, d+1) \cup\{z\})$ is a $d$-polytope whose boundary complex is isomorphic to $\partial \Sigma$, demonstrating sufficiency. (In fact, $P$ is $n$-stacked in the sense of [7].)

A sketch of the construction of $\Sigma$ follows. The case $H(1)=0$ is easily dealt with. For $H(1) \geqslant 1$, let $U=\left\{u_{1}, \ldots, u_{\nu^{\prime}}\right\}$ where $\nu^{\prime}=H(1)+2 n$. Let $\Psi^{\prime}$ be the set of all $2 n$-subsets $W^{\prime}$ of $U$ of the form $\left\{u_{i_{1}}, u_{i_{1}+1}\right\} \cup \cdots \cup\left\{u_{i_{n}}\right.$, $\left.u_{i_{n}+1}\right\}$ where $1 \leqslant i_{1}, i_{n}+1 \leqslant \nu^{\prime}$, and $i_{j+1}>i_{j}+1,1 \leqslant j \leqslant n-1$. Let $V^{\prime}=$ $\left\{v_{1}, \ldots, v_{d+1-2 n}\right\}, V=V^{\prime} \cup U$ and $\Psi$ be the set of all $(d+1)$-subsets $W$ of $V$ of the form $V^{\prime} \cup W^{\prime}$ for $W^{\prime} \in \Psi^{\prime}$. Give the elements of $\Psi$ the lexicographic linear order $<$ induced by $u_{1}<\cdots<u_{v^{\prime}}$. Let $\Phi_{n}$ be the set of all monomials in the variables $Y_{1}, \ldots, Y_{s}$ of degree at most $n$, where $s=H(1)$. A one-to-one order preserving correspondence $\beta: \Phi_{n} \rightarrow \Psi$ can be defined. From $\Phi_{n}$ choose the lexicographic order ideal of monomials $M$ associated with $(H(0), \ldots, H(n))$. List the elements of $M$ in order $m_{1}<\cdots<m_{\mu}$. Consider the corresponding elements of $\Psi, F_{i}=\beta\left(m_{i}\right)$. Let $\Sigma$ be the $d$-dimensional simplicial complex whose maximal simplices are $F_{1}, \ldots, F_{\mu}$. It can be shown that $\Sigma$ is shellable with 
shelling order $F_{1}, \ldots, F_{\mu}$ and that $h^{(d+1)}(\Sigma)=(H(1), \ldots, H(d+1))$.

Relabel the elements of $V=\left\{v_{1}, \ldots, v_{d+1-2 n}, u_{1}, \ldots, u_{\nu^{\prime}}\right\}$ as $\left\{v_{1}\right.$, $\left.\ldots, v_{\nu}\right\}$ where $\nu=H(1)+d+1$. Consider the cyclic polytope $C(\nu, d+1)=$ $\operatorname{conv}\left\{v_{1}, \ldots, v_{\nu}\right\}$ where $v_{i}=\left(t_{i}, t_{i}^{2}, \ldots, t_{i}^{d+1}\right) \in \mathbf{R}^{d+1}, t_{1}<\cdots<t_{\nu}$. This notation implicitly defines a one-to-one correspondence between $V$ and the vertex set of $C(\nu, d+1)$. Then $\left\{F_{1}, \ldots, F_{\mu}\right\}$ is a representation of a shellable proper collection $B$ of facets of $C(\nu, d+1)$. The existence of a realization of $C(\nu, d+1) \subseteq \mathbf{R}^{d+1}$ and of a point $z \in \mathbf{R}^{d+1}$ beyond precisely the facets in $B$ reduces to finding rational numbers $t_{1}<\cdots<t_{\nu}$ satisfying a finite number of polynomial inequalities. This can be accomplished by an application of a version of Tarski's Principle (see e.g. [2, Theorem 13, p. 290]). Once this is done, the desired simplicial polytope $P$ can be obtained as previously described.

A PROBleM OF KLEE ON UPPER BOUNDS. For $3 \leqslant d \leqslant r<\nu$, a polytope (resp. spherical complex) $P$ is of type $(d, \nu, r)$ if $P$ is a $d$-polytope (resp. $(d-1)$ spherical complex) with $\nu$ vertices, one of which is incident to precisely $r$ edges. The problem, stated by Klee in a dual fashion, is to determine $\max f_{d-1}(P)$ over all simplicial polytopes $P$ of type $(d, \nu, r)$. Klee places bounds on this number and determines it in some particular cases [3], [4]. We offer the complete solution with the following

THEOREM 2. Let $S$ be a simplicial sphere of type $(d, v, r)$. Then $f_{i}(S) \leqslant$ $f_{i}(C(\nu-1, d))+f_{i}(C(r+1, d))-f_{i}(C(r, d)), 0 \leqslant i \leqslant d-1$. Further, there exists a simplicial d-polytope $P^{*}$ that satisfies all of the above expressions with equality. (Here $f_{i}(C(d, d))$ is 2 if $i=d-1$, and is $f_{i}(C(d, d-1))$ otherwise.)

The bounds are established in the same manner that Stanley uses in [8], relying on the fact that $h$-vectors of simplicial spheres are 0 -sequences. $P^{*}$ is obtained from a construction similar to that used in the proof of Theorem 1. Here, however, the desired polytope is $\operatorname{conv}(C(\nu-1, d) \cup\{z\})$ for an appropriate $z$. By a triangulation argument similar to that of pulling vertices of polytopes it can in fact be shown that $P^{*}$ achieves the maximum number of $i$-dimensional faces over the class of all (not necessarily simplicial) spherical complexes of type $(d, v, r)$. (Spherical complexes are defined in [6].)

\section{REFERENCES}

1. B. Grünbaum, Convex polytopes, Wiley, New York, 1967.

2. N. Jacobson, Lectures in abstract algebra, Vol. III, Van Nostrand, Princeton, N. J., 1964.

3. V. Klee, Polytope pairs and their relationship to linear programming, Acta Math. 133 (1974), 1-25.

4. Convex polyhedra and mathematical programming, Proc. Internat. Congr. Mathematicians, Vol. I, Vancouver, 1974, pp. 485-490.

5. P. McMullen, The numbers of faces of simplicial polytopes, Israel J. Math. 9 (1971), 559-570.

6. P. McMullen and G. C. Shephard, Convex polytopes and the upper bound conjecture, London Math. Soc. Lecture Note Ser. 3, Cambridge, 1971. 
7. P. McMullen and D. W. Walkup, A generalized lower-bound conjecture for simplicial polytopes, Mathematika 18 (1971), 264-273.

8. R. Stanley, The upper bound conjecture and Cohen-Macaulay rings, Studies in Appl. Math. 54 (1975), 135-142.

9. Cohen-Macaulay complexes, Higher combinatorics, M. Aigner (ed.), D. Reidel, Dordrecht, 1977, pp. 51-62.

10. Hilbert functions of graded algebras, Advances in Math. 28 (1978), $57-83$.

SCHOOL OF OPERATIONS RESEARCH AND CENTER FOR APPLIED MATHEMATICS, CORNELL UNIVERSITY, ITHACA, NEW YORK 14853 
